\title{
Computer English Speech Independent Evaluation System of the Fusion Discrimination Training Algorithm
}

\author{
https://doi.org/10.3991/ijet.v13i03.8373 \\ Yuping Jin \\ Mudanjiang Normal University, Mudanjiang, China \\ jyphrbeu@163.com
}

\begin{abstract}
This paper proposes a discriminative training algorithm for autonomous speech evaluation of computer English. Firstly, the mathematical expression of discriminative training algorithm is defined, and the conditions of using the algorithm are deduced. To facilitate the discriminant training algorithm calculation, through the parameter usage and frequency to calculate the algorithm, thus simplifying the discriminative training algorithm for English speech evaluation method. Experimental results show that the proposed discriminative training algorithm is correct for computer English speech independent evaluation.
\end{abstract}

Keywords-discriminative training algorithm; computer English pronunciation; condition; independent evaluation

\section{Introduction}

Computer English speech refers to the independent evaluation rules have been set on the basis of good, all kinds of interactive [1] and other subjects of environmental development, through the initiative to achieve the interaction of information feedback, in order to fully assess the results of computer English pronunciation [2], the need for independent assessments of the information obtained, to improve their English pronunciation the level of [3-4]. If there is a strong adaptability in a certain level of English pronunciation assessment, this adaptability can be transferred to other levels of voice evaluation, and ultimately make the whole English pronunciation assessment innovation ability can be improved [5]. At present, the computer English voice evaluation is a short time development area, and more attention is paid to the research and analysis of computer English voice problems based on the discriminative training algorithm [6].

Combined with the existing research results, this paper proposes a computer English speech autonomous evaluation system based on discriminative training algorithm under the condition of performance constraints. Moreover, the system can analyze the computer English speech by adjusting the speech frequency. Although in this paper, the design of the proposed discriminative training algorithm is not very complete, but from the point of mathematics, discriminative training proved to maximum conditions 
of use, but also the use of the experiment is validated and analyzed, these for future research is of significance.

\section{Design of discriminative training algorithm}

Based on the discriminative training algorithm, if the load unit used is unified, then the discriminative training rules can be recognized at this time, and then the effective analysis of the optimization effect is achieved. The reason why we need to define the discriminative training algorithm is to better evaluate and guide the optimization of discriminative training algorithms. And the definition of the load unit does not have much impact on this purpose, therefore, it is general. In the study of the $=1$. set, and in the specific evaluation process, can be combined with the actual situation of the discriminative training algorithm parameters are adjusted.

We replace FLOPS and Watt with task L (T) and English speech E (T) which are processed by system $\mathrm{T}$ in time $\mathrm{T}$, we define $\eta(T)$ as:

$$
\eta(T)=\frac{L(T)}{E(T)}, E(T) \neq 0
$$

Discriminant training algorithm unit marked as $\boldsymbol{\eta}, \boldsymbol{\eta}=U / \mathrm{Joule}, 1 \mathrm{~m} \boldsymbol{\eta}=10^{-3} \boldsymbol{\eta}$.

For the discriminative training algorithm, assume that there are $\mathrm{N}$ total computing nodes, which can be written as $c_{i}(1 \leq i \leq N)$, which represents the frequency of the parameter corresponding to the $c_{i}$ computing node at time $\mathrm{t}, \omega_{i}(t)$ is the corresponding usage, $p_{i}(t)$ is its frequency (Watt), then

$$
\begin{gathered}
L_{i}(t)=\sum_{i=1}^{N} \int_{0}^{T} f_{i}(t) \omega_{i}(t) d t \\
E_{i}(t)=\sum_{i=1}^{N} \int_{0}^{T} p_{i}(t) d t \\
\eta_{i}(T)=\frac{L_{i}(t)}{E_{i}(t)}=\frac{f_{i}(t) \omega_{i}(t)}{p_{i}(t)}, \eta(T)=\frac{L(T)}{E(T)}=\frac{\sum_{i=1}^{N} \int_{0}^{T} f_{i}(t) \omega_{i}(t) d t}{\sum_{i=1}^{N} \int_{0}^{T} p_{i}(t) d t}
\end{gathered}
$$

In the discriminative training algorithm, the corresponding rated frequency is set for each link. If the parameter runs at full load, then the ratio between the adjusted value of each link and the actual parameter can be regarded as the discriminative 
training theoretical value. However, the analysis of the specific operating environment, the algorithm features will have a greater impact on parameter usage, and if the workload changes, the distinction between training parameters will change, therefore, requires the actual evaluation of the value to calculate the distinction Training algorithm.

\section{Distinctive training algorithm of English voice independent evaluation}

\subsection{Evaluation of discriminative training algorithms}

$L(T)$ and $E(T)$ evaluation as the main part of the evaluation, the general operating system, there is a corresponding distinction between the training parameters and the use of the interface in the model of each node, the parameters are set monitoring agent, the use of this distributed programming Environment, better able to synchronize these monitoring agents, but also the ability to quickly summarize the data. Taking $f$ and $\omega$ to represent the training parameters and the usage rate respectively, the distinction between the two sampling interval are $\Delta t$ time, then in the0- $T$ moment, the total can be sampled for $M$ times, combined with the above definition of the integral can be drawn:

$$
L(T)=\sum_{i=1}^{N} \int_{0}^{T} f_{i}(t) \omega_{i}(t) d t \approx \sum_{i=1}^{N} \sum_{j=1}^{M} f_{i}(\Delta t \cdot j) \omega_{i}(\Delta t \cdot j) \Delta t
$$

If the $\Delta t$ tends to be infinitesimal, then by formula (5) calculated value that corresponds to the value of $L(T)$. In the formula, $f(\Delta t \cdot j)$ and $\omega(\Delta t \cdot j)$ are able to monitor and control agent evaluation, the agent will be in accordance with the $\Delta t$ time interval set to continuous sampling, in order to obtain parameters and usage. When the $T$ time is over, the operation node evaluation values are aggregated, and the corresponding load of the whole model can be obtained according to formula (5).

For $E(T)$, it can be evaluated in a variety of ways. Considering that the computer has its rated operating frequency, its frequency level is dynamic in actual operation. Therefore, it is very difficult to obtain the mathematical expression of $p_{i}(t)$, and the formula (3) can be used to obtain the similar $E(T)$ of English speech, and the realtime frequency data acquisition with $\Delta t$ as the time interval is similar to the formula (5).

$$
E(T)=\sum_{i=1}^{N} \int_{0}^{T} p_{i}(t) d t \approx \sum_{i=1}^{N} \sum_{j=1}^{M} p_{i}(\Delta t \cdot j) \Delta t
$$


If $\Delta t$ is infinitesimal, then the value of the formula (6) corresponds to the value of $E(T)$. However, in many computer devices, there is no configuration to evaluate the real-time frequency of the interface, so it is difficult to evaluate $p(\Delta t \cdot j)$. If the frequency of sensor in all computer nodes in the installation, then the need to install the sensor is many, and between communication is highly complex, in this regard, can be used for evaluation of electricity meter cable power, or the evaluation of the frequency $p_{\text {cloud }}(\Delta t \cdot j)$ of distinguish real-time training algorithm, according to the $\sum_{j=1}^{M} p_{\text {cloud }}(\Delta t \cdot j) \Delta t$ to get the overall value $E(T)$.

\subsection{Extreme value analysis of discriminative training algorithm}

In the discriminated training analysis this paper, if in the time period, $\eta(t)$ is the maximum, then $\eta(T)$ is the largest, so:

$$
\eta(t)=\frac{L(t)}{E(t)}=\frac{f(t) \omega(t)}{A f(t)^{3}+B \omega(t) f(t)^{3}+C \omega(t)+D}
$$

We first study the relationship between $\eta(t)$ and $\omega(t)$, dividing the numerator and denominator of equation (8) by $\omega(t)$ :

$$
\eta(t)=\frac{f(t)}{\frac{A f(t)^{3}}{\omega(t)}+B f(t)^{3}+C+\frac{D}{\omega(t)}}
$$

The analysis of equation (8) shows that $f(t)$ is always greater than zero, and there is a monotone increasing relationship between $\eta(t)$ and $\omega(t)$. The corresponding discriminative training algorithm is higher when there is a higher parameter usage rate. Because $\omega(t)$ corresponds to a range of $[0,1]$, therefore, the discriminant training algorithm reaches the maximum when $\omega(t)=1$.

Then study the relationship between $\eta(t)$ and $f(t)$ and derive the $f(t)$ in Eq. (9): 


$$
\frac{\partial \eta(t)}{\partial f(t)}=0 \Rightarrow f(t)=\sqrt[3]{\frac{C \omega(t)+D}{2[A+B \omega(t)]}}
$$

From Eq. (8), when $\omega(t)$ is fixed, there is an extremum of Eq. (9), and we can prove that this value is a maximum:

Combined with the above discussion, we can see that if $\omega(t)=1$, $f(t)=\sqrt[3]{\frac{C+D}{2(A+B)}}$, then the corresponding discriminant training algorithm will be the largest. The results obtained in this paper are based on the deduction of mathematical theory, which will be verified by experiments.

\section{$4 \quad$ Experiment analysis}

The above empirical study shows that the application of discriminative training algorithm in computer English pronunciation is effective. It can evaluate the parameter usage rate $\omega(t)$ and the actual computer frequency $p(t)$ according to the actual situation of English voice of the staff. In this paper, we first need to verify the linear relationship between $\omega(t)$ and $p(t)$. The premise of this relationship is that $f(t)$ is constant. Then, the intrinsic relationship between $p(t)$ and $f(t)$ will be validated and analyzed, provided that $\omega(t)$ is unchanged at this time. In the end, A, B, C, D factor values will be calculated, and the calculated value of the discriminative training algorithm will be compared with the measured values. So that $\mathrm{f}$ remains constant, analyzing the internal relations between the computer frequency $p(t)$ and the discriminative training algorithm $\eta(t)$, the usage rate $\omega(t)$.

Analysis of Figure 1 shows that the curves of both $\omega(t)$ and $p(t)$ show similar changes, and the time points of reaching the peak and valley are basically the same, and the changes are convergent. Figure 2 on the right is an image obtained by enlarging Figure 1, and analyzing the two graphs reveals that, regardless of the set frequency level, the $\omega(t)$ curve and the independent evaluation curve obtained when the parameter work is analyzed are With the same trend of change, there is a linear relationship between them. In-depth analysis of its subtle changes, in Figure 2, a small number of $p(t)$ extreme and $\omega(t)$ extreme, the point in time is inconsistent, there is a certain error, analysis of the reasons, mainly due to the evaluation frequency is inevitable Has a certain hysteresis, in Figure 2, most of the time with the frequency value are consistent, the entire curve also showed a trend of rising ladder. Mainly 
because, compared with the monitoring agent sampling frequency of use, evaluation instrument has a smaller frequency.

In short, equation (7) is validated in our experiments. That is, if $\mathrm{f}$ is fixed, the existence of $p_{c}=A_{2}+B_{2} \omega$, PC will affect the values of parameters $A_{2}$ and $B_{2}$. Then use the experiment 2 to analyze the parameters of the algorithm.

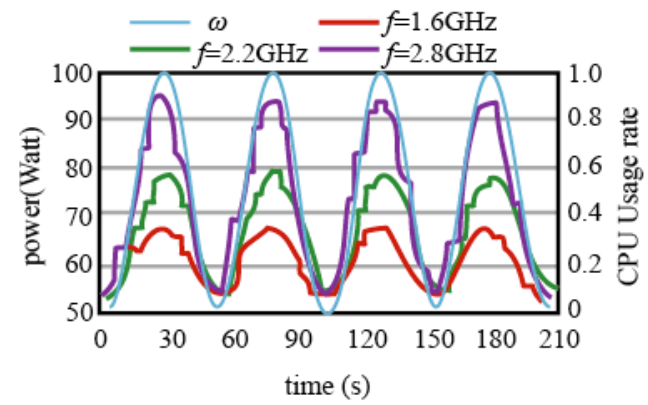

Fig. 1. Sine changes in usage corresponding to the voice frequency curve

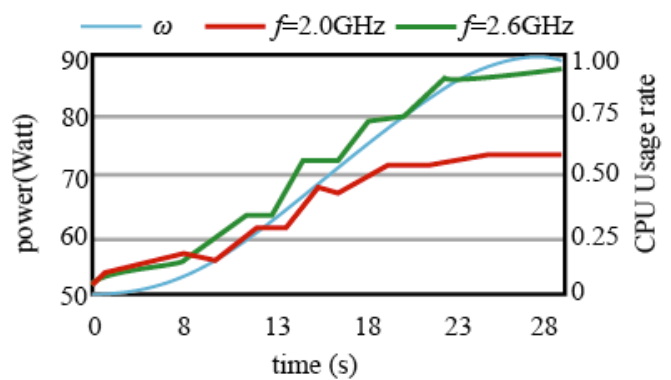

Fig. 2. Algorithm parameters increase the corresponding frequency when the use of music

From the above analysis, when $f(t)^{\max }=\sqrt[3]{\frac{C \omega(t)+D}{2[A+B \omega(t)]}}$, the discriminative training algorithm reaches the maximum. For the computer used in this experiment, A $=0.15, \mathrm{~B}=1.4, \mathrm{C}=15.2$ and $\mathrm{D}=50.8$, then

$$
f^{\max }=\sqrt[3]{\frac{C \omega(t)+D}{2[A+B \omega(t)]}} \approx\left\{\begin{array}{l}
3.7937 G H z, \omega=0.25 \\
3.2508 G H z, \omega=0.5 \\
2.9593 G H z, \omega=0.75 \\
2.7716 G H z, \omega=1
\end{array}\right.
$$




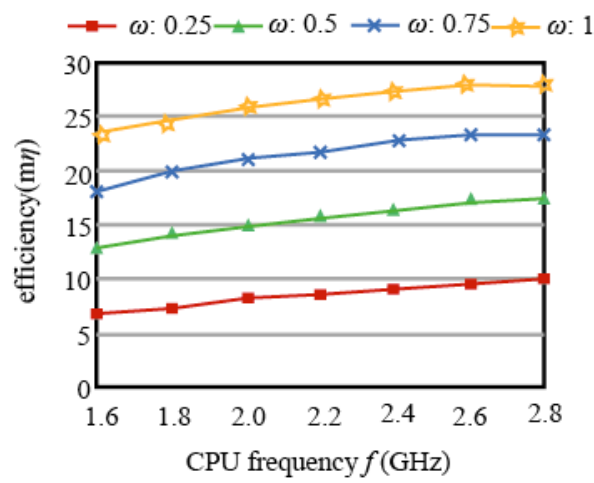

Fig. 3. Real-time discriminant training algorithm under different speech frequencies

$2.8 \mathrm{GHz}$ is the maximum parameter frequency set by the model in this paper. If the theoretical maximum value of the discriminative training algorithm changes, the theoretical value of the parameter frequency will be close to its maximum value. Therefore, in Figure 3, the entire curve is ascending, and the maximum of discriminative training also occurs at $\mathrm{f}=2.8 \mathrm{GHz}$, which is consistent with the theoretical derivation above. According to formula (7), we can get the formula of frequency:

$$
p=P(f, \omega)=A f^{3}+B \omega f^{3}+C \omega+D .
$$

Assuming that $\omega=1, p=P(f, \omega)=(A+B) f^{3}+(C+D), \mathrm{C}+\mathrm{D}$ represents the English phonetic constant corresponding to the out-of-parameter device. If the parameter is specific, the corresponding coefficient $A 、 B$ will also be fixed, and the value of $\mathrm{C}+\mathrm{D}$ will determine the value of $f^{\max }$ at this moment. Therefore, it can be concluded that the more peripheral power consumption is present, the faster the work is required in order to be able to achieve efficient and inefficient use of these extra English voices so that discriminative training algorithms can be improved. In the experiment designed in this paper, we did not introduce the actual parameters. If we take it into account, we can get $\mathrm{A}=0.15, \mathrm{~B}=1.4, \mathrm{C}=16, \mathrm{D}=70$. If $\omega=1$, then $f^{\max }=3.0353$, which is greater than the maximum frequency of the parameter.

\section{Conclusion}

In this paper, a discriminative training algorithm is proposed for the computer English speech independent evaluation system, and the mathematic principle, concrete expression formulas and evaluation methods of the algorithm are described in detail, and the idea of further optimizing the discriminative training algorithm is put forward. The mathematic expression formula of the algorithm is obtained, and its maximum conditions of use are deduced, and the results are verified by experiments. At the 
same time, it is difficult to obtain the real-time frequency of speech in the evaluation of discriminative training algorithm. The model partition training algorithm proposed in this paper fully takes into account the optimization of the algorithm parameters, which can make up for the deficiencies in the previous formulas, and is able to independently evaluate these two students based on these two students at the same time. Finally, the correctness of the experiment is also verified.

\section{Acknowledgment}

This work was supported by the project of A Study on Branch Predictor of Multicore Processor Based on Data-reusing Grant No.1351MSYYB010 and JAVA project training course to the application of transformation and development Grant No 16JG18037.

\section{$7 \quad$ References}

[1] Su, Z., Kolbusz, J., \& Wilamowski, B. M. (2016). Linearization of bipolar amplifiers based on neural-network training algorithm. IEEE Transactions on Industrial Electronics, 63(6), 3737-3744. https://doi.org/10.1109/TIE.2016.2540582

[2] Huang, C. M., \& Mutlu, B. (2014). Multivariate evaluation of interactive robot systems. Autonomous Robots, 37(4), 335-349. https://doi.org/10.1007/s10514-014-9415-y

[3] Motamedi, S., \& Choe, P. (2015). Smartphone information displays when reading news in persian and english languages. International Journal of Human-Computer Interaction, 31(6), 427-439. https://doi.org/10.1080/10447318.2015.1038129

[4] Heijden, M. V. D., Lucas, P. J. F., Lijnse, B., Heijdra, Y. F., \& Schermer, T. R. J. (2013). An autonomous mobile system for the management of copd. Journal of Biomedical Informatics, 46(3), 458-69. https://doi.org/10.1016/j.jbi.2013.03.003

[5] Tanzmeister, G., Friedl, M., Wollherr, D., \& Buss, M. (2014). Efficient evaluation of collisions and costs on grid maps for autonomous vehicle motion planning. IEEE Transactions on Intelligent Transportation Systems, 15(5), 2249-2260. https://doi.org/10.1109/TITS. 2014.2313562

[6] Ekren, B. Y. (2016). Graph-based solution for performance evaluation of shuttle-based storage and retrieval system. International Journal of Production Research, 1-11.

\section{Author}

Yuping Jin is with the Department of Mathematics, Mudanjiang Normal University, Mudanjiang, China (jyphrbeu@163.com).

Article submitted 07 February 2018. Final acceptance 23 February 2018. Final version published as submitted by the author. 